\title{
Pushing the Frontiers of First-Principles Based Computer Simulations of Chemical and Biological Systems
}

\author{
Elizabeth Brunk, Negar Ashari, Prashanth Athri, Pablo Campomanes, F. Franco de Carvalho, \\ Basile F. E. Curchod, Polydefkis Diamantis, Manuel Doemer, Julian Garrec, Andrey Laktionov, \\ Marco Micciarelli, Marilisa Neri, Giulia Palermo, Thomas J. Penfold, Stefano Vanni, Ivano Tavernelli, \\ and Ursula Rothlisberger*
}

\begin{abstract}
The Laboratory of Computational Chemistry and Biochemistry is active in the development and application of first-principles based simulations of complex chemical and biochemical phenomena. Here, we review some of our recent efforts in extending these methods to larger systems, longer time scales and increased accuracies. Their versatility is illustrated with a diverse range of applications, ranging from the determination of the gas phase structure of the cyclic decapeptide gramicidin S, to the study of G protein coupled receptors, the interaction of transition metal based anti-cancer agents with protein targets, the mechanism of action of DNA repair enzymes, the role of metal ions in neurodegenerative diseases and the computational design of dye-sensitized solar cells. Many of these projects are done in collaboration with experimental groups from the Institute of Chemical Sciences and Engineering (ISIC) at the EPFL.
\end{abstract}

Keywords: Anti-cancer - DNA repair - Excited states · First-principles molecular dynamics · Force matching · GPCR · Gramicidin · Nonadiabatic dynamics · Prion · QM/MM simulations · Solar cells ·

Time-dependent density functional theory

\section{Introduction}

Recent developments in the computational sciences introduce the possibility of studying a diverse range of biologically relevant problems in chemistry and physics. In particular, first-principles Molecular Dynamics (MD) ${ }^{[1,2]}$ has become a very successful and widespread method for the study of chemical and biochemical reactions in complex environments. ${ }^{[3]}$ However, despite the rapid advances of computer simulations, numerous limitations continue to encumber their application to biological phenomena, in which restrictions are mainly due to tractable system size, accessible time window/sampling and limited accuracy.

Since its foundation in 2002, the Laboratory of Computational Chemistry

${ }^{\star}$ Correspondence: Prof. U. Rothlisberger Laboratory of Computational Chemistry and Biochemistry

Ecole Polytechnique Fédérale de Lausanne EPFL $\mathrm{CH}-1015$ Lausanne

Tel.: +41216930325

Fax: +41216930320

E-mail: ursula.roethlisberger@epfl.ch and Biochemistry has made efforts to overcome these hurdles. To address the system size problem, we have been active in the development of mixed Quantum Mechanical/ Molecular Mechanical (QM/ MM) $M D$ methods, ${ }^{[4,5]}$ which provide a means to study chemical reactions in light of the protein environment. While considering the entire protein in an ab initio MD framework would require an exorbitant amount of computational resources, a mixed QM/MM framework overcomes this limitation by describing only the subset of catalytically active atoms by quantum mechanics and the rest of the systems at the more expedient level of a classical force field. In addition, the development of nonadiabatic MD based on time-dependent density functional theory (TDDFT) ${ }^{[6-9]}$ and its combination with a QM/MM framework has opened a new avenue for first-principles studies of the dynamics of molecular systems in electronically excited states, as it offers a computationally affordable way for the calculation of excited potential energy surfaces (PESs), atomic forces, and nonadiabatic couplings as a functional solely of the ground state density (or equivalently of the corresponding Kohn-Sham orbitals).

Besides various enhanced sampling techniques, ${ }^{[10-12]}$ improvements in simulation times can be achieved via force matching approaches, in which atomic forces obtained for the QM subsystem in a QM/MM setup are used to enhance the accuracy of classical force fields.

In addition, the development of Dispersion Corrected Atom-Centered Potentials (DCACPS $)^{[13-16]}$ has improved dramatically the description of weakly bound compounds (van der Waals complexes) within Density Functional Theory (DFT) calculations (in full quantum as well as QM/MM setups).

Over the years, our group has been active in developing $\mathrm{QM} / \mathrm{MM}$ and $a b$ initio MD methods, such as these, and we have used the advancements of these approaches to embrace a wide range of study for a variety of biological systems, varying in size from less than a hundred atoms, such as a drug molecule, to more than a hundred thousand atoms, such as a lipid membrane protein, and time scales, extending from attoseconds to microseconds.

\section{Methods}

Extending System Size: Mixed Quantum Mechanical/Molecular Mechanical Simulations in Ground and Excited States

A complete description of a chemical reaction can often not be obtained if its environment is neglected and it is well known that solvent molecules, cell membranes or protein matrices can have a large effect on potential energy surfaces. In our approach, 
we separate the global system into two distinct parts: an active part (described at the DFT or Linear Response (LR)-TDDFT level of theory) and a classically described environment. Such a partitioning is named $Q M / M M$ for Quantum Mechanics/ Molecular Mechanics, highlighting the quantum description of the electrons in the active part and the corresponding classical approximation to describe the environment.

In our hybrid QM/MM MD approach, ${ }^{[4,5]}$ the total Hamiltonian of a QM/ MM system is

$$
H=H_{Q M}+H_{M M}+H_{Q M / M M}
$$

where $H_{O M / M M}$ describes the interaction between the QM and the MM part. $H_{O M / M M}$, the coupling Hamiltonian, can be divided into a bonded term, if covalent bonds exist between the QM and the MM subsystems, and a non-bonded term (Eqn. (2)) where $\mathbf{R}$ is the position of MM atom $i$ with the point charge $q_{\mathrm{i}}, \rho$ is the total charge density of the quantum system (electronic and ionic), and $v_{v d W}\left(R_{i j}\right)$ is the van der Waals interaction between atoms $i$ and $j$ (see ref. [3] for more details).

In the employed QM/MM scheme, the van der Waals interaction term is simply taken from the classical force field. The Coulomb term is described at the QM level by replacing the classical point charges of the MM atoms by a suitable (smoother) charge distribution. For reasons of numerical efficiency, the electrostatic interaction between the QM system and the more distant MM atoms (outside a certain cutoff distance from any QM atom) is included via a Hamiltonian term explicitly coupling the multiple moments of the quantum charge distribution with the classical point charges. For details of the implementation see refs [3-5].

For QM/MM simulations in an excited electronic state $I$, the ground state electronic density contained in $\rho$ in Eqn. (3) is supplemented by the linear response density $\delta \rho_{\text {el }}^{I}$ (see ref. [17]). QM/MM simulations of excited states open the door for the study of light-induced ultrafast phenomena like non-radiative decays or photochemical reactions.

\section{Including Nonadiabatic Effects}

When excited states are explicitly considered in dynamics, the BornOppenheimer approximation can break down and nonadiabatic effects need to be explicitly incorporated in the nuclear propagation. There are a variety of different methods to serve this purpose. Tully's trajectory surface hopping ${ }^{[18]}$ and Ehrenfest dynamics $^{[19]}$ are among the most widely

$$
H_{\text {nonbonded }}=\sum_{i \in \mathrm{MM}} q_{i} \int d \mathbf{r} \frac{\rho(\mathbf{r})}{\left|\mathbf{r}-\mathbf{R}_{i}\right|}+\sum_{\substack{i \in \mathrm{MM} \\ j \in \mathrm{QM}}} v_{v d W}\left(R_{i j}\right)
$$

used. These two methods require ground and excited state potential energy surfaces, the forces on the nuclei, ${ }^{20]}$ and nonadiabatic coupling terms. We have shown that LR-TDDFT can be used for the calculation of nonadiabatic coupling vectors. ${ }^{[6-8]}$ Therefore, LR-TDDFT is our electronic structure method of choice for nonadiabatic dynamics.[21-23] This approach has been validated by numerous applications, for example the isomerization of excited protonated formaldimine, ${ }^{[22,24]}$ the photochemical ring-opening of oxirane, ${ }^{[25]}$ laserinduced excitations of small molecules ${ }^{[26]}$ with future extension to local control theory, or ultrafast relaxation of ruthenium (II) trisbipyridine in water ${ }^{[17]}$ (see ref. [9] for more examples).

\section{Towards Longer Time Scales: On the Fly Force Matching}

Recently, we have developed an automated on-the-fly force matching approach for the parameterization of classical force fields from QM/MM data. ${ }^{[27]}$ This method uses a QM/MM trajectory to extract the quantum forces acting on the different atoms. These are then used as a reference for eters through the minimization of a penalty function based on the difference between the quantum and the classical forces

$$
\sigma^{2}=\sum_{l=1}^{L} \sum_{i \in Q M}\left\|F_{l i}^{M M}-F_{l i}^{Q M}\right\|^{2}
$$

where the index $l$ runs over all selected configurations along the QM/MM trajectory.

We applied force matching to the parameterization of a new anticancer azole-bridged platinum compound binding to DNA. [28] Since large-scale conformational changes cannot be observed during QM/MM MD runs (due to the short maximal time scale the optimization of the force field param- of few tens of ps), classical MD simulations are mandatory to capture the slow (ns- $\mu$ s time scale) distortions of the DNA helix (Fig. 1).

Despite the success of the forcematching scheme for classical force fields parameters, in some cases one cannot completely ignore the quantum character of the electronic degrees of freedom. For this reason, we are currently working on a force-matching protocol for the on-thefly parameterization of a semi-empirical quantum Hamiltonian that can be used to simulate biochemical reactions with sufficient sampling times to explore the chemical space effectively during slow conformational rearrangements in macromolecules.

\section{Increasing the Accuracy of DFT: Dispersion Corrected Atom Centered Potentials}

The inability to capture weak interactions (London forces) is known to be one of the significant flaws of DFT based on approximate exchange correlation functionals. ${ }^{[29,30]}$ Recently, we proposed a new method, Dispersion-Corrected AtomCentered Potentials (DCACP), that enables a highly accurate description of dispersion interactions when conventional DFT is used. ${ }^{[13]}$ These atom-centered potentials that correct standard generalized gradient approximation (GGA) functionals have a simple analytic form, identical to the nonlocal part of the atomic pseudopotentials developed by Goedecker et al. ${ }^{[31]}$ as shown in Eqn. (4) where $r=\left|r-R_{\mathrm{I}}\right|$ is the distance from the electron to the position of the nucleus $I$ and the normalized projector, embraced by spherical harmonics,

\section{$p_{l}(r) \propto r^{l} \exp \left[-r^{2} /\left(2 \sigma_{2}^{2}\right)\right]$}

is centered on the position of nuclei $I$. In the current version of the DCACP library

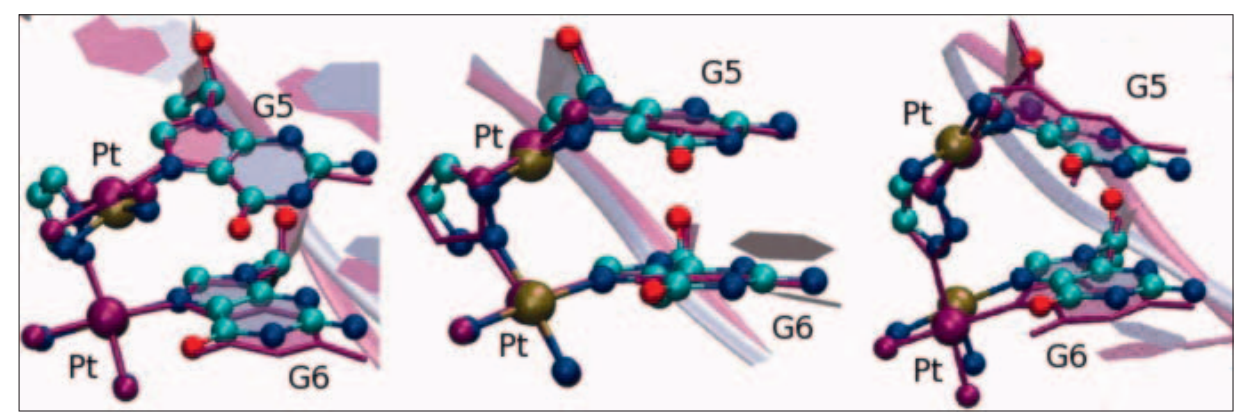

Fig. 1. Superposition of average QM/MM (purple) and classical forced-matched MD structures of three dinuclear Pt-drug-DNA complexes. Force-matched classical MD structure is in ball and stick and $\mathrm{QM} / \mathrm{MM}$ structure in sticks for the drug-DNA moiety. 


$$
\hat{v}_{l}^{D C A C P}\left(r, r^{\prime}\right)=\sum_{l=0}^{l_{\max }} \sum_{m=-1}^{+l} Y_{l m}(\hat{r}) p_{l}(r) \sigma_{1} p_{l}\left(r^{\prime}\right) Y_{l m}^{*}\left(\hat{r}^{\prime}\right)
$$

we obtain accurate results using only one projection channel $(1=3)$. Inclusion of more channels leads to the improvement of the tail of the interaction curve. ${ }^{[14]}$ Using DCACPs greatly improves the interactions of base stacking and hydrogen bonding in biomolecules ${ }^{[15]}$ and the DCACP approach significantly improves the BLYP description of liquid water both for structural (e.g. radial pair correlation functions) and dynamical (e.g. self-diffusion coefficient and orientational correlation times) properties. ${ }^{[16]}$

\section{Applications}

In the following, we briefly report some applications of the methods described above. These examples demonstrate the range of possibilities offered by these approaches in the study of biological and chemical processes both in the gas phase and in complex environments (modeled at a classical molecular mechanical level or at a quantum mechanical level).

\section{Benchmarking the Performance of Computational Methods: Gas Phase Structure of Gramicidin S}

Gramicidin S (GS) is a cyclic decapeptide that acts as a naturally occurring antibiotic. ${ }^{[32,33]}$ In this project, high-level theory was used in conjunction with cold-ion spectroscopy performed by the group of $\mathrm{T}$. Rizzo at the EPFL to solve the conformerselective gas-phase structure of GS. ${ }^{[34]}$

On the computational side, we performed extended MD simulations to sample the configurational space. The exploration was guided by structural restraints derived from electrospray ionization mass spectrometry, and laser spectroscopy at ul- tracold temperatures. The structures isolated by this directed search were optimized using DFT, and frequencies of the lowest energy structure were confirmed to be in good agreement with the infrared spectrum of the most abundant conformer. ${ }^{[35]}$ As far as we know, gramicidin $\mathrm{S}$ exemplifies the largest molecule whose gas-phase structure has been solved.

\section{Prion Protein and Amyloid $\beta$ Peptides}

Neurodegenerative diseases, such as Parkinson's, Huntington's, Alzheimer's and Creutzfeldt-Jakob disease, are characterized by the formation of protein plaques in the brain, resulting from the accumulation and aggregation of insoluble proteins. As a consequence, the neurons in the central nervous system are seriously damaged.[36] Moreover, the plaques contain certain metal ions, which seem to be crucial to the onset of the disease. ${ }^{[37,38]}$

We have studied two proteins and their complexes with transition metal ions, which are involved in the development of these diseases, namely the Prion Protein $\operatorname{PrP}^{\mathrm{c}}$ and the amyloid $\beta$ peptide $\mathrm{A} \beta 16$.

$\mathrm{PrP}^{\mathrm{c}}$ is the cellular prion protein, whose misfolded form $\left(\mathrm{PrP}^{\mathrm{Sc}}\right)$ aggregates, leading to the development of a number of neurodegenerative diseases. We have investigated the binding of $\mathrm{Cu}$ (II), ${ }^{[39]} \mathrm{Mn}$ (II) and $\mathrm{Zn}(\mathrm{II})^{[40]}$ ions to the C-terminal structured domain of mouse $\mathrm{PrP}^{\mathrm{c}}$. We were able to localize the preferred metal binding sites and suggest a possible role of metal ions in metal assisted protein aggregation.

Alzheimer's disease is brought about by the aggregation of beta amyloid $(A \beta)$ peptides, the metabolites of the amyloid precursor protein. The amyloid plaques are known to contain large amounts of $\mathrm{Cu}$ ions. Even though $\mathrm{A} \beta-\mathrm{Cu}$ complexes have been studied extensively, ${ }^{[41-45]}$ there are some aspects that remain unclear, such as the exact nature of the $\mathrm{Cu}$ coordination sphere. To shed light on these issues, we are working in collaboration with the experimental group of $\mathrm{H}$. Girault at the EPFL to study $\mathrm{A} \beta 16$ and $\mathrm{A} \beta 16-\mathrm{Cu}(\mathrm{I})$ in gas phase with classical as well as QM/MM simulations (Fig. 2).

\section{Metallo Anti-Cancer Drugs}

The field of anti-cancer metallodrugs has been traditionally dominated by classical drugs like cisplatin $\left[\mathrm{Pt}(\mathrm{Cl})_{2}\left(\mathrm{NH}_{3}\right)_{2}\right]$. The mode of action of these compounds relies on their binding to DNA, thus blocking the metabolism of rapidly dividing cancer cells, but also inflicting damage on healthy cells, causing undesirable side effects. Therefore, non-classical chemotherapeutic strategies have gained significant prominence by targeting the specific cellular signaling pathways that cancer cells depend on for growth, proliferation, metastasis, and angiogenesis. ${ }^{[46,47]}$ One of the preferential targets for non-classical anticancer drugs is the Glutathione S-Transferase (GST) P1-1 enzyme (Fig. 3 ), which is often found over-expressed in solid tumors. Its main function is the detoxification of toxic compounds in the cytoplasm. In addition, GST P1-1 also regulates the mitogen-activated protein (MAP) pathway, involved in cellular survival and cell death signaling. Several organometallic GST inhibitors showing anti-tumoral properties have been recently synthesized in the group of P. Dyson at the EPFL and the corresponding GST pro-drug adducts have been characterized. ${ }^{[48,49]}$ However, in spite of the wealth of structural information provided by the

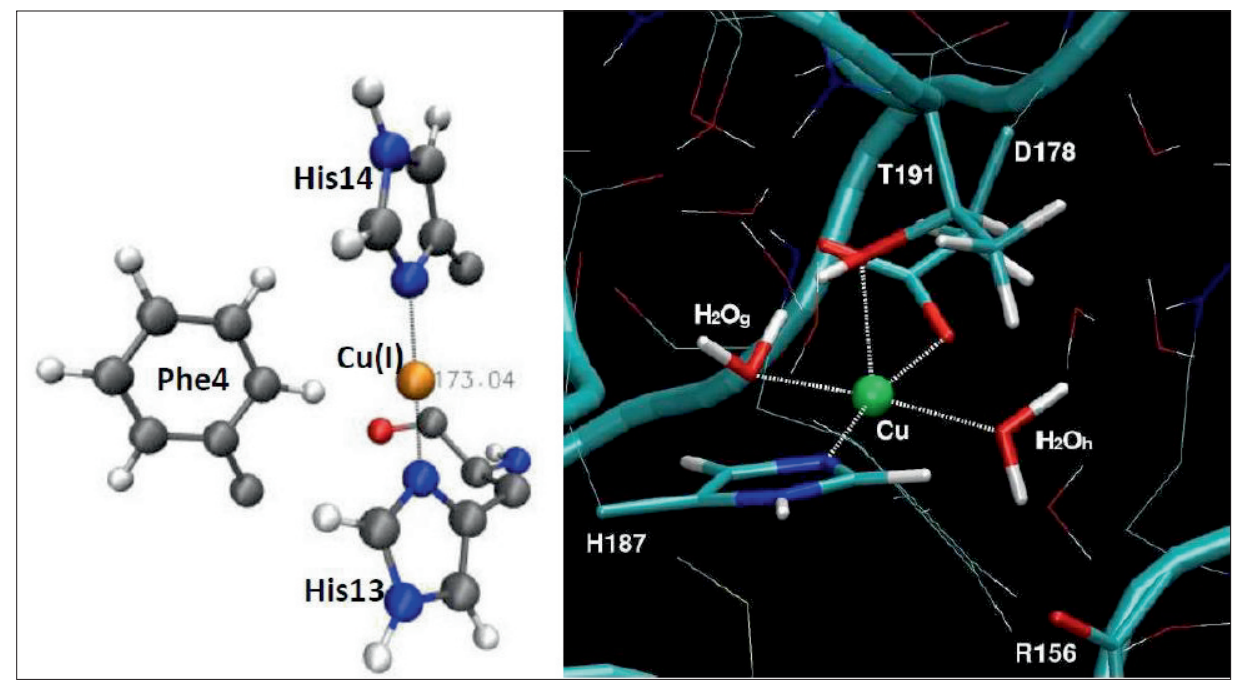

Fig. 2. Binding Site of $\mathrm{Cu}(\mathrm{I})$ in $\mathrm{A} \beta 16$ (gas phase, left), and a $\mathrm{Cu}(\mathrm{II})$ binding site in mouse PrP (right).

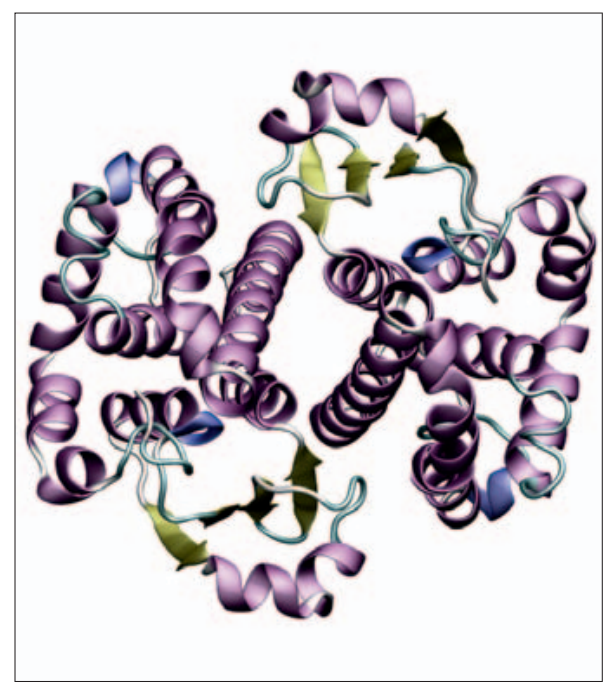

Fig. 3. Glutathione S-Transferase P1-1. 
X-ray structures, many details about the mode of action of these drugs are still unknown. To gain the knowledge required to guide further experimental investigations and assist the rational development of more effective and targeted anticancer drugs, we are currently investigating both the sequence of events involved in the inhibitor-enzyme recognition process, and the activation mechanism caused by the covalent binding of the pro-drug to its target enzyme by means of a mixed approach based on classical and QM/MM MD simulations.

\section{DNA Repair Enzymes}

The integrity of intracellular DNA is constantly being challenged by various forms of stress such as reactive oxygen species, produced during aerobic respiration, or from the environment in the form of ultraviolet radiation. As a result of oxidation, single or double-strand lesions arise in the DNA molecule in addition to oxidative transformations, which affect the nucleotide bases or sugar phosphates. Base excision repair glycosylases have recently been identified as the class of enzymes that provide the main form of defense against most types of lesions in DNA, including oxidation of guanine residues into radical 7,8-dihydro-8-oxoguanine, alkylation, hydration, deamination and cyclo-addition in pyrimidine dimers. ${ }^{[50]}$

Despite intense studies, ${ }^{[51-53]}$ the catalytic mechanisms of many DNA repair enzymes are still not established. Using a hybrid QM/MM scheme, as detailed in the methods section, allows for the investigation of chemical reactions such as these, in which a complex environment must be taken into consideration. Using this framework has enabled the study of the DNA repair enzyme endonuclease IV and the ultrafast process involving the DNA repair photolyase splitting reaction of the UV-induced thymine dimer.[54,55] The catalytic mechanism of the MutY glycosylase enzyme is now being investigated to elucidate the structural features of the ordered water molecules in the active site and their contribution to the catalytic mechanism. ${ }^{[55,56]}$

\section{Signal Transduction in G Protein Coupled Receptors}

$\mathrm{G}$ protein coupled receptors (GPCRs) are a large eukaryotic protein family of transmembrane receptors that react to a signal coming from the outside of the cell to generate a cellular response through the activation of a signal transduction pathway mediated by a heterotrimeric $\mathrm{G}$ protein. The function of these receptors is regulated via the binding of endogenous or exogenous ligands that can either lead to activation (agonists) or inac-

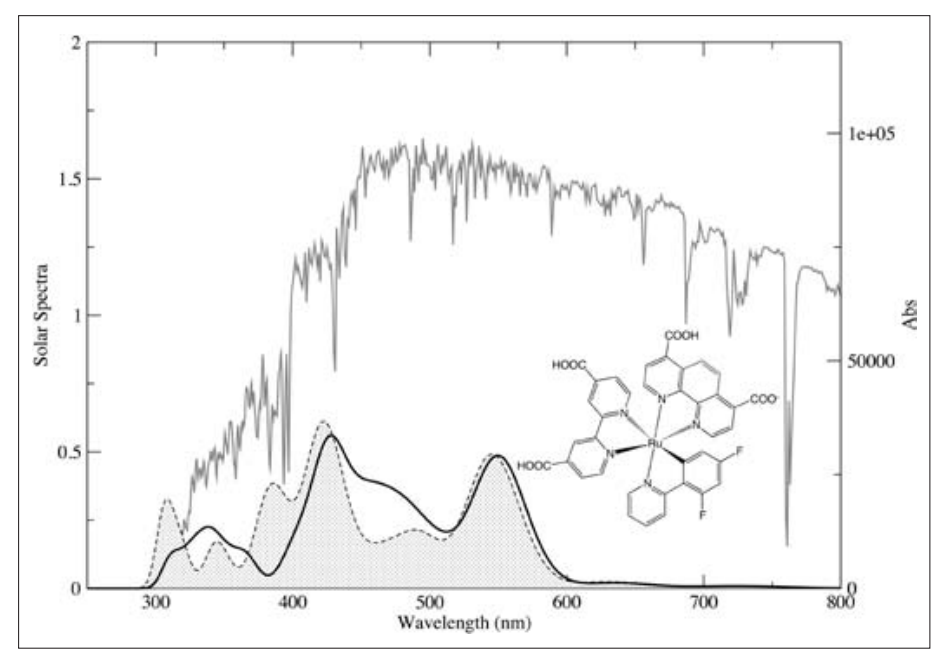

Fig. 4. Example of the absorption spectrum (black thick line) of a dye (structure on the right) (M06/6311G*, LANL2DZ for $\mathrm{Ru}$, implicit solvent). For comparison, the computed absorption spectrum of a standard dye is given ${ }^{[64]}$ (dashed line and gray area), together with the solar spectrum at ground (AM 1.5) (gray).

tivation (inverse agonists or antagonists). However, only recently, determination of the crystal structures of diffusible ligands GCPRs, the $\beta 2$ and $\beta 1$ adrenergic receptors ( $\beta$ ARs)bound to inverse agonists/antagonists, has provided an atomic view of the ligand binding mode ${ }^{[57,58]}$ as well as of the overall three-dimensional structure.

Using force field based classical MD, we have followed the $\mu$ s time evolution of $\beta$ ARs under different external conditions. Within this approach, the non-native modifications induced by the different engineering techniques used for crystallization have been identified ${ }^{[59]}$ and some of the mechanisms through which these receptors have managed to optimize their function through evolution have been described and quantified. In detail, this has led to suggest a possible agonist binding mode to $\beta \mathrm{ARs}^{[60]}$ and to identify crucial micro-switches during receptor activation, ${ }^{[61]}$ also in comparison with the evolutionary related GPCR, rhodopsin.[62]

\section{Improving the Efficiency of Dye- Sensitized Solar Cells through Computational Dye Design}

LR-TDDFT has become an important tool for the design of new molecules exhibiting tailored spectral properties. Important examples of such systems are dye-sensitized solar cells, ${ }^{[63]}$ whose efficiency relies strongly on absorption features of the adsorbed dye. In collaboration with the group of M. Graetzel at the EPFL, we are using LR-TDDFT to develop a novel library of chemical ligands, which, once complexed to ruthenium, enhance the overall absorption under solar radiation (Fig. 4). ${ }^{[64]}$ Furthermore, we are interested in creating a more realistic environment for the dyes with an explicit description of solvent molecules and titanium dioxide nanoparticles, together with a dynamical description of the injection process.

\section{Conclusion and Outlook}

Computer simulations based on a first-principles quantum mechanical description have reached a level that can provide realistic and predictive descriptions of complex chemical and biological phenomena. With the continuously increasing computer power in combination with new methodological developments such as the ones described here we hope to push the frontiers even further and provide more and more reliable computer experiments as possible guides to experimental efforts.

\section{Acknowledgements}

The authors would like to thank the Swiss NSF for financial support within the NCCR MUST and grant No. 200020-130082 and 200020-116374. The COST action CM0702 is also acknowledged for funding and the CADMOS project for computer time on the IBM Blue Gene/P

Received: June 24, 2011

[1] R. Car, M. Parrinello, Phys. Rev. Lett. 1985, 55, 2471

[2] D. Marx, J. Hutter, in 'Modern Methods and Algorithms of Quantum Chemistry,' Ed. J. Grotendorst, John von Neumann Institute for Computing, Jülich, NIC Series, 1, 2000.

[3] U. Rothlisberger, P. Carloni, Lect. Notes Phys. 2006, 704, 437.

[4] A. Laio, J. VandeVondele, U. Rothlisberger, J. Chem. Phys. 2002, 116, 6941.

[5] A. Laio, J. VandeVondele, U. Rothlisberger, J. Phys. Chem. B 2002, 106, 7300.

[6] I. Tavernelli, E. Tapavicza, U. Rothlisberger, $J$. Chem. Phys. 2009, 130, 124107.

[7] I. Tavernelli, B. F. E. Curchod, U. Rothlisberger, J. Chem. Phys. 2009, 131, 196101.

[8] I. Tavernelli, B. F. E. Curchod, A. Laktionov, U. Rothlisberger, J. Chem. Phys. 2010, 133, 194104.

[9] B. F. E. Curchod, P. Campomanes, A. Laktionov, M. Neri, T. J. Penfold, S. Vanni, I. Tavernelli, U. Rothlisberger, Chimia 2011, 65, 5, 330.

[10] A. Laio, M. Parrinello, PNAS 2002, 99, 12562.

[11] J. VandeVondele, U. Rothlisberger, J. Chem. Phys. 2000, 113, 4863.

[12] J. VandeVondele, U. Rothlisberger, J. Chem. Phys. 2001, 115, 7859. 
[13] O. A. von Lilienfeld, I. Tavernelli, U. Rothlisberger, Phy. Rev. Lett. 2004, 93, 153004.

[14] I. Tavernelli, I.-C. Lin, U. Rothlisberger, Phys. Rev. B 2009, 79, 045106-1-045106-5.

[15] I.-C. Lin, U. Rothlisberger, Phys. Chem. Chem. Phys. 2008, 10, 2730.

[16] I.-C. Lin, A. Seitsonen, M. Coutinho-Neto, I. Tavernelli, U. Rothlisberger, J. Phys. Chem. B 2009, 113, 1127 .

[17] I. Tavernelli, B. F. E. Curchod, U. Rothlisberger, Chem. Phys. 2011, doi:10.1016/j.chemphys. 2011.03.021.

[18] J. C. Tully, J. Chem. Phys. 1990, 93, 1061.

[19] I. Tavernelli, U. F. Röhrig, U. Rothlisberger, Mol. Phys. 2005, 103, 963.

[20] J. Hutter, J. Chem. Phys. 2003, 118, 3928.

[21] I. Tavernelli, Phys. Rev. B 2006, 73, 094204.

[22] E. Tapavicza, I. Tavernelli, U. Rothlisberger, Phys. Rev. Lett. 2007, 98, 023001.

[23] B. F. E. Curchod, I. Tavernelli, U. Rothlisberger, Phys. Chem. Chem. Phys. 2011, 13, 3231.

[24] I. Tavernelli, E. Tapavicza, U. Rothlisberger, $J$. Mol. Struct.-TheoChem 2009, 914, 22.

[25] E. Tapavicza, I. Tavernelli, U. Rothlisberger, C. Filippi, M. E. Casida, J. Chem. Phys. 2008, 129, 124108.

[26] I. Tavernelli, B. F. E. Curchod, U. Rothlisberger, Phys. Rev. A. 2010, 81, 052508.

[27] P. Maurer, A. Laio, U. Rothlisberger, J. Chem. Theor. Comp. 2007, 3, 628.

[28] K. Spiegel, A. Magistrato, P. Maurer, P. Ruggerone, U. Rothlisberger, P. Carloni, J. Reedijk, M. L. Klein, J. Comput. Chem. 2007, 29, 38.

[29] Y. Zhao, D. Truhlar, J. Chem. Theory Comput. $2005,1,415$.

[30] E. Hohenstein, S. Chill, C. Sherrill, J. Chem. Theory Comput. 2008, 4, 1996.

[31] S. Goedecker, M. Teter, J. Hutter, Phys. Rev. B 1996, 54, 1703.

[32] G. N. Tishchenko, V. I. Andrianov, B. K Vainstein, M. M. Woolfson, E. Dodson, Acta Crystallogr. Sect. D 1997, 53, 151.

[33] E. J. Prenner, R. N. A. H. Lewis, R. N McElhaney, Biochim. Biophys. Acta Biomembr. 1999, 1462, 201.
[34] N. S. Nagornova, M. Guglielmi, M. Doemer, I. Tavernelli, U. Rothlisberger, T. R. Rizzo, O. V. Boyarkin, Angew. Chem. Int. Ed. 2011, 50, 1.

[35] O. V. Boyarkin, S. R. Mercier, A. Kamariotis, T. R. Rizzo, J. Am. Chem. Soc. 2006, 128, 2816.

[36] D. M. Skovronsky, V. M.-Y. Lee, J. Q. Trojanowski, Annu. Rev. Pathol. Mech. Dis. 2006, $1,151$.

[37] P. Zatta, R. Lucchini, S. J. van Rensburg, A. Taylor, Brain Res. Bull. 2003, 62, 15 .

[38] A. I. Bush. Curr. Opinion Chem. Biol. 2000, 4, 184.

[39] M. C. Colombo, J. VandeVondele, S Van Doorslaer, A. Laio, L. Guidoni, U. Rothlisberger, Proteins 2008, 70, 1084.

[40] M. C. Colombo, 'On the Structural Stability of the Free and Metal-loaded C-terminal domain of the Prion Protein', Ph.D Thesis 2006, 3, Lausanne, EPFL, no 3592.

[41] A. B. Baumktner, S. L. Bernstein, T. Wyttenbach, G. Bitan, D. B. Teplow, M. T. Bowers, J.-E. Shea, Protein Science 2006, 15, 420.

[42] Y. Lu, M. Prudent, L. Qiao, M. A. Mendez, H. H. Girault, Metallomics 2010, 2, 474.

[43] A. S. Bush, Trends Neurosci. 2003, 26, 207.

[44] R. A. Himes, G. Y. Park, G. S. Siluvai, N. J. Blackburn, K. D. Karlin, Angew. Chem. Int. Ed. 2008, 47, 9084

[45] L. Guilloreau, L. Damian, Y. Coppel, H. Mazarguil, M. Winterhalter, P. Faller, J. Biol. Inorg. Chem. 2006, 11, 1024.

[46] J. S. Sebolt-Leopold, J. M. English, Nature 2006, 441, 457

[47] W. H. Ang, P. J. Dyson, Eur. J. Inorg. Chem. 2006, 20, 4003 .

[48] W. H. Ang, L. J. Parker, A. De Luca, L. Juillerat-Jeanneret, C. J. Morton, M. Lo Bello, M. W. Parker, P. J. Dyson, Angew. Chem. Int. Ed. 2009, 48, 3854.

[49] L. J. Parker, L. C. Italiano, C. J. Morton, N. C. Hancock, D. B. Ascher, J. B. Aitken, H. H. Harris, P. Campomanes, U. Rothlisberger, A. De Luca, M. Lo Bello, W. H. Ang, P. J. Dyson, M. W. Parker, Chem. Eur. J. 2011, in press.

[50] G. Slupphaug, B. Kavli, H. E. Krokan, Mutation Research 2003, 531, 231.
[51] J. C. Fromme, A. Banerjee, S. J. Huang, G. L. Verdine, Nature 2004, 427, 652.

[52] J. Stivers, Y. L. Jiang, Chem. Rev. 2003, 103.

[53] P. J. Berti, J. A. B. McCann, Chem. Rev. 2006 106, 506.

[54] F. Masson, T. Laino, I. Tavernelli, U. Rothlisberger, J. Hutter, J. Am. Chem. Soc. 2008, 130, 3443.

[55] D. Bucher, F. Masson, S. Arey, U. Rothlisberger, in 'Quantum Biochemistry', 2010, Ed C. F. Matta, Wiley-VCH Verlag GmbH \& Co. KGaA, Weinheim, Germany.

[56] E. Brunk, S. Arey, I. Tavernelli, U. Rothlisberger, in preparation.

[57] V. Cherezov, D. M. Rosenbaum, M. A. Hanson, S. G. Rasmussen, F. S. Thian, H. J. Choi, P. Kuhn, W. I. Weis, B. K. Kobilka, R. C. Stevens, Science 2007, 318, 1258.

[58] T. Warne, M. J. Serrano-Vega, J. G. Baker, R. Moukhametzianov, P. C. Edwards, R. Henderson, A. G. W. Leslie, C. G. Tate, G. F. X Schertler, Nature 2008, 454, 486.

[59] S. Vanni, M. Neri, I. Tavernelli, U. Rothlisberger, Biochemistry 2009, 48, 4789.

[60] S.Vanni, M.Neri, I. Tavernelli, U. Rothlisberger, PLOS Computational Biology 2011, 7, e1001052.

[61] S. Vanni, M. Neri, I. Tavernelli, U. Rothlisberger, J. Mol. Biol. 2010, 397, 1339.

[62] M. Neri, S.Vanni, I. Tavernelli, U. Rothlisberger, Biochemistry 2010, 49, 4827.

[63] B. O'Regan, M. Grätzel, Nature 1991, 335, 737.

[64] T. Bessho, E. Yoneda, J.-H. Yum, M. Guglielmi, I. Tavernelli, U. Rothlisberger, H. Imai, M. K Nazeeruddin, M. Grätzel, J. Am. Chem. Soc 2009, 131, 5930 . 